\title{
Pop III stars and the earliest phases of the evolution of galaxies and IGM
}

\author{
Francesca Matteucci ${ }^{1}$, Silvia Ballero ${ }^{1}$, Francesco Calura ${ }^{1}$, Cristina \\ Chiappini $^{2}$, Antonio Pipino ${ }^{1}$ \\ ${ }^{1}$ Dipartimento di Astronomia, Universita' di Trieste, Via R. Bazzoni, 3, 34124 Trieste, Italy
email: matteucci@ts.astro.it \\ ${ }^{2}$ Osservatorio Astronomico di Trieste, INAF, Via G.B. Tiepolo 11, 34124 Trieste, Italy
}

\begin{abstract}
We discuss the effects of very massive Population III stars on the chemical evolution of the Milky Way, elliptical galaxies and the intergalactic medium (IGM) at high reshift. It is shown that the effects produced by Pop III stars on the early evolution of the most common chemical abundances $(\mathrm{C}, \mathrm{N}, \mathrm{O}, \alpha$-elements, $\mathrm{Fe})$ are negligible if these stars formed only for a very short period of time, corresponding to the suggested threshold metallicity $\left(Z_{t h r} \sim 10^{-4} Z_{\odot}\right)$. For a higher threshold metallicity and therefore a longer period of time, the predicted results are at variance with observations. It is also concluded that the IGM at high redshift $(z=5.0)$ cannot have been enriched only by very massive Pop III stars, but that the contribution of lower mass stars is necessary. The same conclusion holds for DLA systems at high redshift.
\end{abstract}

Keywords. Stars: abundances, galaxies: evolution

\section{Introduction}

Many theoretical and observational facts suggest the possible existence of massive and very massive Population III (zero metallicity, hereafter Pop III) stars. First of all the Jeans mass should have been larger in the early stages of galaxy evolution due to its dependence on the gas temperature. Metals, in fact, are the most effective coolant in gas clouds and they allow for the formation of small mass protostar. So, in absence of metals, the formation of very massive stars should be favoured. Hydrodynamical simulations of the collapse and fragmentation of the primordial gas clouds suggest that the very first stars should have masses larger than $100 M_{\odot}$ (Bromm \& Larson 2004) . This high mass biased star formation must have lasted until a critical threshold metallicity was reached in the gas. The latest calculations constrain this value of $Z_{t h r}$ in the range $\left(10^{-6}-10^{-4} \cdot Z_{\odot}\right)$ (Schneider et al. 2002). Observationally, a reason to invoke Pop III stars resides in the recent results by WMAP experiment which have provided constraints on the number of ionizing photons produced by very massive stars at redshifts z $>15$ (Cen 2003). Also the metals observed in the Lyman- $\alpha$ forest at $\mathrm{z}=4-5$ suggest an early field stellar population which has enriched the IGM (Schaye et al. 2003). Finally the fact that no stars with zero metallicity has ever been observed can sustain the idea of a very massive early stellar generation. The most metal poor star known is $\mathrm{HE}-1327-2326$ with $[\mathrm{Fe} / \mathrm{H}]=-5.4 \pm 0.2$ (Frebel et al. 2005). Therefore we would like to answer to the following questions: do we need an early generation of very massive Pop III stars to explain the abundance patterns in the most metal poor stars observed? Which are the effects on the predicted chemical abundances if we assume that for a short period of time only massive and very massive stars formed? To this purpose we have tested several sets of yields computed for Pop III stars by means of chemical evolution models following in detail the evolution of several chemical elements $(\mathrm{C}, \mathrm{N}, \mathrm{O}, \alpha$-elements, $\mathrm{Fe})$. In particular we tested the effects 
of these yields on the evolution of the Galaxy and of a typical elliptical galaxy. The same yields have also been used to compute the chemical enrichment expected in the high redshift IGM by a generation of very massive stars. Very massive stars $\left(M>100 M_{\odot}\right)$ are interesting objects from the evolutionary point of view. They die as Pair-CreationSupernovae (PCSNe): pair-creation instability sets in during the H- and He- core burning phases inducing core collapse, explosive O-burning and SN explosion. They do not leave any remnant. Nucleosynthesis in PCSNe has been computed since the early eighties (e.g. Ober et al. 1983) and more recently by Heger \& Woosley (2002) (HW02) and Umeda \& Nomoto (2002) (UN02). Chieffi \& Limongi (2002), and Chieffi \& Limongi (2004) (CL) also provided yields for zero-metallicity stars but in the mass range $10-80 M_{\odot}$. The nucleosynthesis occurring in PCSNe is different from that occurring in less massive stars and with metallicity different from zero. As an example, UN02 found that Pop III stars in the range $30-150 M_{\odot}$ and $>270 M_{\odot}$ implode as black holes without contributing to any chemical enrichment while HW02 found that Pop III stars with $M>260 M_{\odot}$ and between 40 and $140 M_{\odot}$ become black holes. Therefore, only the range of masses between $140-150 M_{\odot}$ and $260-270 M_{\odot}$ can contribute to the early chemical enrichment. CL found non negligible production of $\mathrm{C}$ and $\mathrm{N}$ (primary) from Pop III stars with masses $M \leqslant 80 M_{\odot}$.

In the next sections we will describe the chemical evolution models adopted and the results obtained for the Milky Way, an elliptical galaxy and the IGM.

\section{Chemical evolution models}

\subsection{Model for the Milky Way}

We have computed the chemical evolution of the MW by means of the two-infall model of Chiappini et al. (1997) in the latest version of Chiappini et al. (2003). This model assumes that the formation of the halo-thick disk and thin-disk were disentangled and occurred through two distinct gas accretion episodes on quite different timescales (much longer for the thin disk). This model reproduces the majority of the properties of the solar neighbourhood and the whole disk. In our basic model we assumed that at the beginning only massive $\left(M>10 M_{\odot}\right)$ and very massive $\left(M>100 M_{\odot}\right)$ Pop III stars formed until $Z_{g a s}=Z_{t h r}=10^{-4} Z_{\odot}$. We explored also other cases up to $Z_{t h r}=0.1 Z_{\odot}$. The adopted IMF has a slope $\mathrm{x}=1.7$ in the mass range $10-270 M_{\odot}$ for $Z \leqslant Z_{t h r}$ and becomes a Scalo (1986) IMF in $0.1-100 M_{\odot}$ for $\mathrm{Z}>Z_{t h r}$.

\subsection{The model for elliptical galaxies}

For ellipticals we adopted the model of Pipino \& Matteucci (2004) which assumes that ellipticals form by means of a short and intense burst of star formation, occurring at high redshift, followed by a galactic wind. The development of a galactic wind is due to the energy injection from SNe in the interstellar medium (ISM). For this model we adopted a Salpeter (1955) IMF $(\mathrm{x}=1.35)$, which better reproduces the properties of ellipticals, both for Pop III stars and normal stars in the mass range $0.1-100 M_{\odot}$. This model has been tested on real galaxies and reproduce the majority of their chemical and photometric properties.

\section{Results}

\subsection{The Milky Way}

In Figure 1 we show the results obtained by running models for the solar vicinity where PCSNe are forming up to a given $Z_{t h r}=Z_{\text {PopIII }}$. In the left panel of Figure 1 we report 

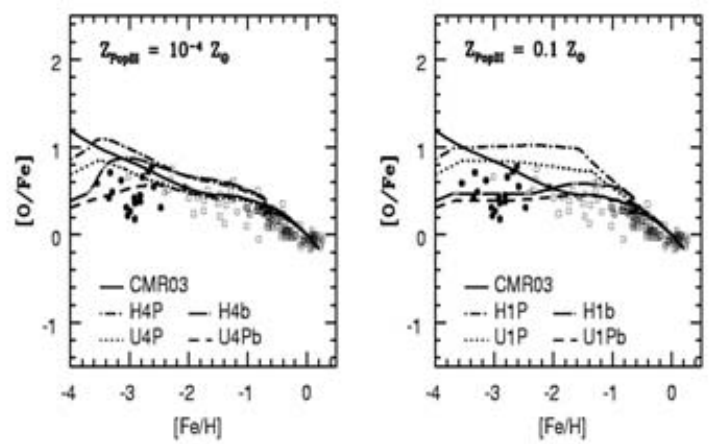

Figure 1. Comparison between model predictions for the $[\mathrm{O} / \mathrm{Fe}]$ vs. $[\mathrm{Fe} / \mathrm{H}]$ in the solar vicinity and data. The models including PCSNe with yields from various sources are compared with the standard model of Chiappini et al. (2003) (CMR03, continuous line) adopting the yields of Woosley \& Weaver (1995) for a solar chemical composition in the range of massive stars.The models labelled $\mathrm{H}$ refer to the yields of HW02, whereas those labelled U refer to the yields of UN02. The label $\mathrm{P}$ indicates the presence of PCSNe, while $\mathrm{b}$ indicates that we considered the black hole formation in the suggested mass ranges. The data for EMPS are from Cayrel et al. (2004) while the others are a compilation from different sources (see François et al. (2004)). Figure adapted from Ballero et al. (2005).

the results for the $Z_{P \text { орIII }}=10^{-4} Z_{\odot}$, whereas in the right panel of the same figure we show an unrealistic case in which the formation of PCSNe occurs until $Z_{P \text { PoIII }}=0.1 Z_{\odot}$. As one can easily see, in the first case the effects of PCSNe, for all the studied yields, on the chemical abundances of extremely metal poor stars (EMPS) are hardly noticeable, whereas in the second case PCSNe produce, in the majority of the cases, results at variance with the observations. For the EMPS we have taken the very accurate data from Cayrel et al. (2004). All models with PCSNe in Figure 1 produce a minimum halo metallicity of $[\mathrm{Fe} / \mathrm{H}]=-4.0$ dex, higher than the most metal poor star known (Frebel et al. 2005) and this fact, by itself, argues against the existence of such very massive stars. The same result is obtained if we assume $Z_{P \text { opIII }}=10^{-6} Z_{\odot}$ and holds also for the other $\alpha$-elements and carbon.

In Figure 2 we show the effects of only massive Pop III stars (no PCSNe) on the [C/Fe] vs. $[\mathrm{Fe} / \mathrm{H}]$ relation with the yields of $\mathrm{CL}$ which produce big differences especially in the $\mathrm{C}$ and $\mathrm{N}$ production.

As one can see from Figure 2, the yields of CL for Pop III stars up to $80 M_{\odot}$ always produce a $[\mathrm{C} / \mathrm{Fe}]$ ratio too high in the EMPS. For the other yields the conclusion is that it is difficult to choose among different models when $Z_{P \text { opIII }}=10^{-4} Z_{\odot}$, whereas they can be discarded if $Z_{P o p I I I}=0.1 Z_{\odot}$. It is worth noting that without PCSNe the minimum metallicity reached in the halo is $[\mathrm{Fe} / \mathrm{H}]=-6.5$ dex.

Finally in Figure 3 we show the $[\mathrm{N} / \mathrm{Fe}]$ vs. $[\mathrm{Fe} / \mathrm{H}]$ which shows how the EMPS have almost solar $[\mathrm{N} / \mathrm{Fe}]$ ratios for the whole metallicity range, thus challenging the secondary nature of $\mathrm{N}$ in massive stars. In Figure 3 are reported the predictions of the standard model which largely underestimate the $\mathrm{N}$ abundance in EMPS. Only models assuming primary $\mathrm{N}$ being constantly produced by massive stars during the halo phase, such as the model proposed by Matteucci (1986) with "ad hoc" primary N and shown for comparison. In the figure we show also the predictions obtained with the yields for Pop III stars by CL. They seem to improve largely the agreement relative to the standard model but only 

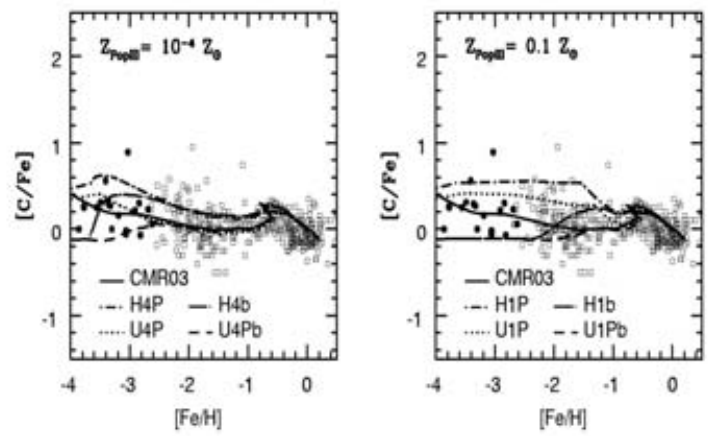

Figure 2. Comparison between model predictions for the $[\mathrm{C} / \mathrm{Fe}]$ vs. $[\mathrm{Fe} / \mathrm{H}]$ in the solar vicinity. The standard model is labelled like in figure 1. Models labelled $\mathrm{C}$ refer to the yields of CL. The labels $\mathrm{M}$ and $\mathrm{Mb}$ mean the absence of PCSNe and the absence of PCSNe plus the black hole formation, respectively. Figure adapted from Ballero et al. (2005).

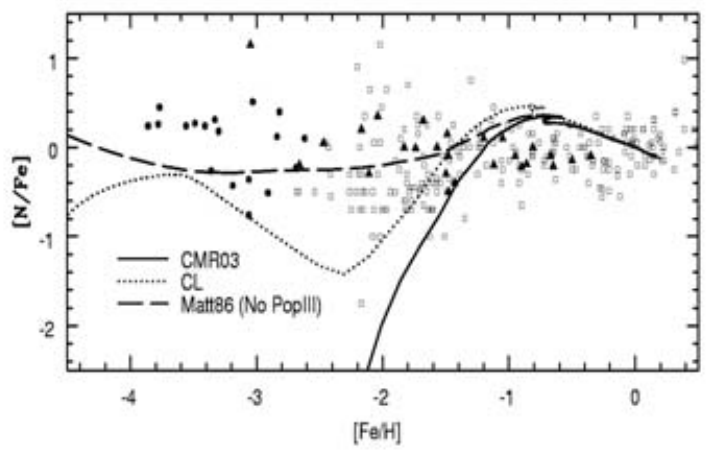

Figure 3. Comparison between model predictions for the $[\mathrm{N} / \mathrm{Fe}]$ vs. $[\mathrm{Fe} / \mathrm{H}]$ in the solar vicinity. The standard model is labelled like in figure 1. The Model labelled CL refers to the yields of Chieffi \& Limongi (2002), Chieffi \& Limongi (2004), whereas Matt86 refers to the model of Matteucci (1986) (described in text). Figure adapted from Ballero et al. (2005).

in the very early phases. The conclusion is that we need a continuous source of primary $\mathrm{N}$ from massive stars (see Chiappini et al. (2005), for a possible solution).

\subsection{Ellipticals}

Matteucci \& Pipino (2005) computed several models for a typical elliptical galaxy with a luminous mass of $10^{11} M_{\odot}$. They included PCSNe with the yields of HW02 and UN02. They assumed different $Z_{P \text { opIII }}$ values as for the Milky Way. Their main result is that the inclusion of even one or two generations of PCSNe alter the following chemical evolution of the ellipticals (they evolve on time scales much below $1 \mathrm{Gyr}$ ), producing too red and too metallic objects besides inducing galactic winds at very early stages. On the other hand, the average predicted $\left.[<M g / F e\rangle_{*}\right]$ of the dominant stellar population is acceptable (in the observed range of 0.2-0.3 dex, Thomas et al. (2002), if the phase of PCSNe has a duration of a few million years. Therefore, the conclusion is that to model these galaxies we do not need PCSNe. In fact, these very massive stars, if existed, were likely to be pregalactic stars and had affected the chemical enrichment of the high redshift IGM. 


\subsection{PCSNe in the IGM}

Matteucci \& Calura (2005) computed how many PCSNe are necessary to pollute the IGM up to a threshold metallicity $Z_{t h r}=10^{-4} Z_{\odot}$. To do that we adopted the yields of HW02 and UN02 and various IMFs (Salpeter and flatter ones). We used the analytical formulation of the Simple Model, under the assumption that the IGM is a closed-box system and that the instantaneous recycling approximation holds for PCSNe which have very short lifetimes. We found that a number between 110 and 115 PCSNe in $1 M p c^{3}$ are necessary to pollute the IGM up to $10^{-4} Z_{\odot}$. We then checked if it is possible to explain only with PCSNe the $\mathrm{C} / \mathrm{Si}$ ratio observed at $\mathrm{z}=5$ in the IGM $\left(0.57(\mathrm{C} / \mathrm{Si})_{\odot}\right.$, Songaila 2001) and found that at maximum we could get $(0.011-0.013)(C / S i)_{\odot}$, a value far too low in comparison with the observed one. We concluded that massive Pop III and massive and intermediate Pop II stars are necessary to explain the observations. By using the yields of CL (no PCSNe) we could reach a higher C/Si ratio but still too low $\left(\sim 0.2(C / S i)_{\odot}\right)$. Finally, we compared the abundance ratios produced by PCSNe with the same abundance ratios in DLA systems at high redshift and concluded that the contribution of normal massive and intermediate mass stars is necessary to reproduce the abundances of $\mathrm{C}$ and $\mathrm{N}$ in those systems.

\section{Conclusions}

Our conclusions can be summarized as follows:

- PCSNe of Pop III produce negligible effects on the abundance ratios of very metal poor stars in the Galaxy $(-4.0 \leqslant[\mathrm{Fe} / \mathrm{H}] \leqslant-3.0)$ if they formed for a very short time (until $Z_{t h r}=10^{-4} Z_{\odot}$ ).

- With PCSNe no stars with $[\mathrm{Fe} / \mathrm{H}]<-4.0$ dex are predicted to exist, with Pop III stars with masses up to $80 M_{\odot}$, stars with $[\mathrm{Fe} / \mathrm{H}]=-6.5$ dex are predicted to exist but not below this limit (Ballero et al. (2005).

- Pop III stars do not help in solving the $\mathrm{N}$ problem. Primary production of $\mathrm{N}$ is necessary along all the halo phase to explain the data (Chiappini et al. (2005).

- PopIII stars in ellipticals produce in general too red and too metallic objects (Matteucci \& Pipino (2005). The abundances in the IGM at high reshift and in the DLA systems cannot be reproduced only with PCSNe (Matteucci \& Calura (2005).

\section{Acknowledgements}

We would like to acknowledge funds from the italian MIUR (Ministry for the University and Scientific Research) COFIN2003, project N. 2003028039.

\section{References}

Ballero, S.K., Matteucci, F. \& Chiappini, C. 2005, New Astronomy submitted Bromm, V. \& Larson, R.B. 2004, ARA\&A 42, 79

Cayrel, R., Depagne, E., Spite, M., Hill, V., Spite, F., François, P., Plez, B.,Beers, T.C. et al. 2004, A\&SA 416, 1117

Cen, R. 2003, ApJ 591, L5

Chiappini, C., Matteucci, F. \& Gratton, R. 1997, ApJ 477, 765

Chiappini, C., Romano, D. \& Matteucci, F. 2003, MNRAS 339, 63

Chiappini, C., Matteucci, F. \& Ballero, S.K. 2005, $A \mathscr{E} A$ in press

Chieffi, A. \& Limongi, M. 2002, ApJ 577, 281

Chieffi, A. \& Limongi, M. 2004, ApJ 608, 405

François, P., Matteucci, F., Cayrel, R., Spite,M., Spite, F. \& Chiappini, C. 2004, A\&A 421, 613 
Frebel, A., Aoki, W., Cristlieb, N., Ando, H., Asplund, M., Barklem, P.S., Beers, T.C., Eriksson, K., et al. 2005, Nature 434, 871

Heger, A. \& Woosley, S.E. 2002, HW02 ApJ 567, 532

Matteucci, F. 1986, MNRAS 221, 911

Matteucci, F. \& Pipino, A. 2005, MNRAS 357, 489

Matteucci, F. \& Calura, F. 2005, MNRAS 360, 477

Ober, W.W., El Eid, M.F. \& Fricke, K.J. 1983, A \& $A$ A 119, 61

Pipino, A. \& Matteucci, F, 2004, MNRAS 347, 968

Salpeter, E.E. 1955, ApJ 121, 161

Scalo, J. 1986, Fund. Cosmic phys. 11, 1

Schaye, J., Aguirre, A., Kim, T.S., Theuns, T., Rauch, M. \& Sargent, W.L.W. 2003, ApJ 318, 32

Schneider, R., Ferrara, A., Natarayan, P. \& Omukai, K. 2002, ApJ 571, 30

Songaila, A. 2001, ApJ 561, L153

Thomas, D., Maraston, C. \& Bender, R. 2002, Ap\&SS 281, 371

Umeda, H. \& Nomoto, K. 2002, UN02 ApJ 565, 385

Woosley, S.E. \& Weaver, T.A. 1995, ApJ Supll 101, 181 